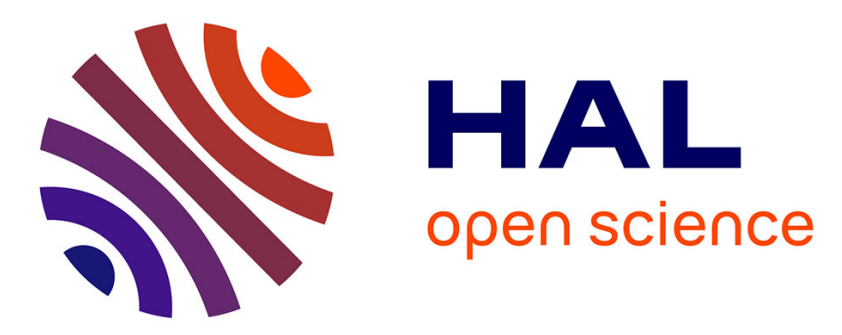

\title{
Simultaneous observations of Jovian quasi-periodic radio emissions by the Galileo and Cassini spacecraft
}

\author{
G. B Hospodarsky, W S Kurth, B Cecconi, D A Gurnett, M L Kaiser, M D
}

Desch, P Zarka

\section{- To cite this version:}

G. B Hospodarsky, W S Kurth, B Cecconi, D A Gurnett, M L Kaiser, et al.. Simultaneous observations of Jovian quasi-periodic radio emissions by the Galileo and Cassini spacecraft. Journal of Geophysical Research Space Physics, 2004, 109 (A9), pp.A09S07. 10.1029/2003JA010263 . hal-03274728

\section{HAL Id: hal-03274728 \\ https://hal.science/hal-03274728}

Submitted on 30 Jun 2021

HAL is a multi-disciplinary open access archive for the deposit and dissemination of scientific research documents, whether they are published or not. The documents may come from teaching and research institutions in France or abroad, or from public or private research centers.
L'archive ouverte pluridisciplinaire HAL, est destinée au dépôt et à la diffusion de documents scientifiques de niveau recherche, publiés ou non, émanant des établissements d'enseignement et de recherche français ou étrangers, des laboratoires publics ou privés. 


\title{
Simultaneous observations of Jovian quasi-periodic radio emissions by the Galileo and Cassini spacecraft
}

\author{
G. B. Hospodarsky, ${ }^{1}$ W. S. Kurth, ${ }^{1}$ B. Cecconi, ${ }^{2}$ D. A. Gurnett, ${ }^{1}$ M. L. Kaiser, ${ }^{3}$ \\ M. D. Desch, ${ }^{3}$ and P. Zarka ${ }^{2}$ \\ Received 30 September 2003; revised 16 January 2004; accepted 23 February 2004; published 29 June 2004
}

[1] The gravity-assist flyby by Cassini of Jupiter on 30 December 2000 and the extended Galileo orbital mission provided a unique opportunity to obtain simultaneous measurements with two spacecraft of many Jovian plasma wave and radio emissions. One of these emissions is Jovian type III radio emissions, also known as Jovian quasi-periodic (QP) emissions. The simultaneous observations of the QP emissions show very similar characteristics, even when the two spacecraft are separated by large distances and located at very different local times (LT). These similarities suggest that this emission is beamed in a strobe light like manner (over a large angular range) and not like a search light rotating with Jupiter's magnetic field, as many other Jovian radio emissions are. The initial source of the QP bursts is likely located near Jupiter. As the emissions propagate through the magnetosphere, the QP bursts appear as enhancements of the trapped continuum. At the magnetosheath the higher density plasma disperses the lower frequency component of the bursts, producing the characteristic "type III like" spectral

shape. INDEX TERMS: 6939 Radio Science: Magnetospheric physics; 5737 Planetology: Fluid Planets: Magnetospheres (2756); 2784 Magnetospheric Physics: Solar wind/magnetosphere interactions; 6984 Radio Science: Waves in plasma; KEYWORDS: Jovian low-frequency radio bursts, quasi-periodic radio bursts, Jupiter radio emissions

Citation: Hospodarsky, G. B., W. S. Kurth, B. Cecconi, D. A. Gurnett, M. L. Kaiser, M. D. Desch, and P. Zarka (2004), Simultaneous observations of Jovian quasi-periodic radio emissions by the Galileo and Cassini spacecraft, J. Geophys. Res., 109, A09S07, doi:10.1029/2003JA010263.

\section{Introduction}

[2] The Jovian magnetosphere is very rich in radio and plasma wave emissions. One interesting radio emission component is the quasi-periodic radio bursts, usually detected in the few $\mathrm{kHz}$ frequency range. These emissions were first detected by Kurth et al. [1989] using the Voyager plasma wave wideband instrument [Scarf and Gurnett, 1977]. These emissions had a dispersive spectral shape similar to solar type III radio bursts, though on a much different timescale, and were given the name Jovian type III radio bursts. The emissions detected by Voyager were impulsive, with durations of about $1 \mathrm{~min}$, were centered on $\sim 10 \mathrm{kHz}$, and ranged from about 5 to $20 \mathrm{kHz}$. The bursts usually had a periodicity of about $15 \mathrm{~min}$, and an event typically lasted 1 or 2 hours. The bursts often showed evidence of a shorter-period structure inside of the main bursts, usually with periods of 2 to $3 \mathrm{~min}$. Figure 1 shows an

\footnotetext{
${ }^{1}$ Department of Physics and Astronomy, University of Iowa, Iowa City, Iowa, USA.

${ }^{2}$ Laboratoire d'Etudes Spatiales et d'Instrumentation en Astrophysique, Observatoire de Paris, Meudon, France.

${ }^{3}$ Laboratory for Extraterrestrial Physics, NASA Goddard Space Flight Center, Greenbelt, Maryland, USA.

Copyright 2004 by the American Geophysical Union. 0148-0227/04/2003JA010263\$09.00
}

example of three of these emissions, with a periodicity of approximately $2 \mathrm{~min}$, that were detected by the Cassini wideband receiver [Gurnett et al., 2004] on 4 October 2000, as Cassini was approaching Jupiter. The examples in Figure 1 show the drifting spectral shape of these bursts and their spectral similarity to solar type III radio bursts, though on a much shorter timescale. The very narrow band emissions that occur at about 1140 at $\sim 20 \mathrm{kHz}$ and at about 1143:40 at $\sim 25 \mathrm{kHz}$ are probably escaping narrow-band electromagnetic radiation from Jupiter [Gurnett et al., 1983].

[3] The Ulysses flyby of Jupiter in 1992 provided the next opportunity to examine these periodic radio emissions. Kaiser et al. [1992] first reported seeing the periodic emissions by the Ulysses/Unified Radio and Plasma Wave (URAP) experiment [Stone et al., 1992]. MacDowall et al. [1993] did a more detailed analysis of the periodic emissions detected by Ulysses and renamed them quasi-periodic (QP) emissions, since Jovian type III designation might imply a specific generation mechanism. The Ulysses observations found that the emissions were made up primarily of two periods, $\sim 15 \mathrm{~min}$ and $\sim 40 \mathrm{~min}$. It should be noted that the sampling period of URAP is usually $144 \mathrm{~s}$, which limits the ability of Ulysses to detect periodic bursts with shorter periods. MacDowall et al. [1993] also found some evidence of a correlation between the occurrence of the QP bursts and the speed of the solar wind, though they suggested that this correlation was probably a proxy for some other solar wind 


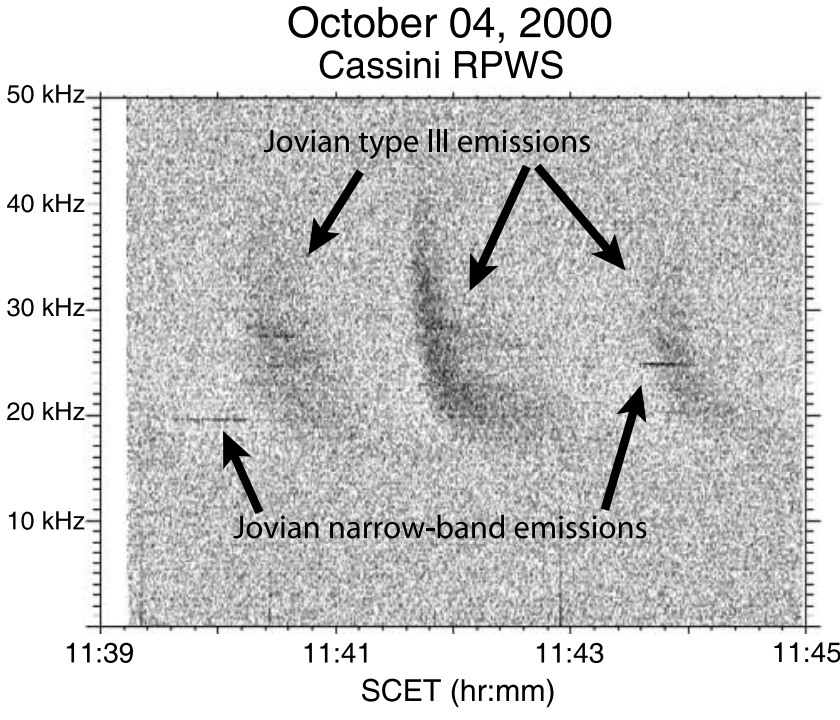

Figure 1. An example of a series of Jovian type III radio bursts (also known as quasi-periodic (QP) bursts) detected by the wideband receiver on Cassini on 4 October 2000, at a distance of $1140 R_{J}$.

parameter. The two periods (labeled QP15 and QP40) were found to have some different characteristics. The QP15 were primarily observed during the inbound part of the Ulysses flyby (low Jovian latitudes). They ranged in frequency from about 5 to $50 \mathrm{kHz}$ and usually had durations of 2 to $5 \mathrm{~min}$. These emissions were predominately observed from $0^{\circ}$ to $140^{\circ}$ Lon $_{\text {III. The }} 40$ min period emissions (QP40) were primarily observed during the outbound part of the flyby (high southern latitudes). The QP40 were only weakly correlated with $\operatorname{Lon}_{\text {III }}\left(\sim 20^{\circ}\right)$ and went to a higher frequency ( 5 to $>200 \mathrm{kHz}$ ) than the QP15. The duration of these emissions ran from 5 to $10 \mathrm{~min}$. The trajectory of Ulysses allowed direction finding of the QP emissions on the outbound part of the flyby. This analysis resulted in the determination of a source region near the Jovian south pole for the QP40 emissions.

[4] The particle instrument on Ulysses [Simpson et al., 1992a] also detected $8 \mathrm{MeV}$ electrons with a similar $40 \mathrm{~min}$ period during the outbound part of the flyby [Simpson et al., 1992b; McKibben et al., 1993]. Some of these electron events were found to be well correlated with the QP40 bursts. However, it is not clear that the $\mathrm{MeV}$ electrons produce the radio emission. Desch [1994] showed that the QP40 bursts were observed at least 5 min before the MeV electrons were detected by Ulysses, suggesting that the radio emission is not produced by the $\mathrm{MeV}$ electrons. However, the observed correlation does suggest that the processes that produce the electrons and the radio waves may be related. MacDowall et al. [1993] also suggested that there was some correlation observed between some QP40 events and $50 \mathrm{keV}$ electrons, though these observations were not discussed in detail. Desch [1994] examined the dispersive shape of the QP bursts and suggested that propagation effects through the Jovian magnetosheath from an initial broadband burst could explain the characteristic spectral shape. However, the source of the initial broadband pulse was not discussed.
[5] Previous examinations of the Galileo plasma wave data [Kurth et al., 1997; Hospodarsky et al., 1998] found that the characteristics of the QP emissions detected by Galileo were more complex than the characteristics reported from the Voyager and Ulysses observations. The first difference between the Galileo observations and the earlier results is that the radio bursts often had multiple (and much more random) periods and were not restricted to only 15 or 40 min periodicities. The period of the QP bursts observed by Galileo ranged from $<1 \mathrm{~min}$ to over $60 \mathrm{~min}$. There are also many intervals where Galileo observes multiple bursts with no obvious periodicity. An examination of one orbit of the Galileo data found no obvious correlation between the occurrence of the QP bursts and the Lon $_{\text {III }}$ [Hospodarsky et al., 1998].

[6] The second major difference from the earlier results is due to the fact that Galileo is usually in the Jovian magnetosphere when the QP bursts are detected. During intervals when Galileo is in the magnetosphere, the QP bursts are usually observed as enhancements of the trapped continuum. Figure 2 shows a typical example of QP emissions detected by Galileo while Galileo is in the Jovian magnetosphere. As can be seen in Figure 2, quasi-periodic enhancements of the trapped continuum are detected by Galileo to much lower frequencies $(\sim 1 \mathrm{kHz})$ than the earlier Ulysses and Voyager observations. This lower-frequency component is below the typical solar wind (and magnetosheath) plasma frequency and therefore could not propagate out of the magnetosphere. Higher-frequency bursts (few tens of $\mathrm{kHz}$ ) are also observed and usually merge with the lower frequency bursts. Menietti et al. [2001] looked at high-resolution wideband measurements obtained by Galileo of the quasi-periodic enhancements of the trapped continuum and found that the broadband bursts did not show the low-frequency dispersion detected by Voyager and Cassini outside the magnetosphere

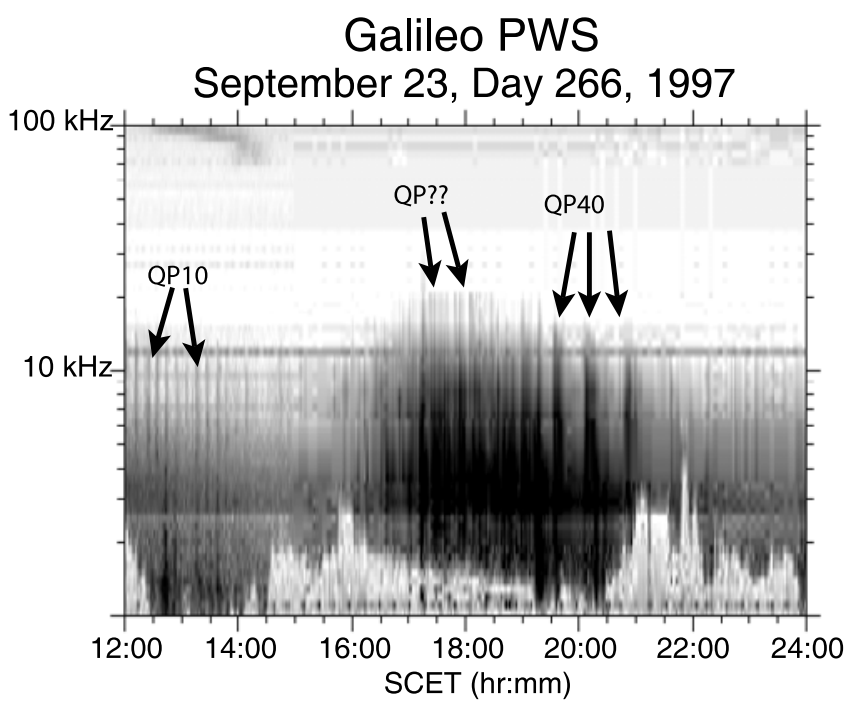

Figure 2. Frequency-time spectrograms showing typical QP bursts detected by Galileo in the Jovian magnetosphere. The QP bursts appear as enhancements of the trapped continuum and often display a wide range of periods, ranging from 1 or $2 \mathrm{~min}$ to $60+\mathrm{min}$. In this example, $\sim 10 \mathrm{~min}$, $\sim 40 \mathrm{~min}$, and somewhat random periods are observed. 
(see Figure 1). This lack of the lower-frequency dispersion when Galileo is in the magnetosphere suggests that the source of the dispersion is propagation through the magnetosheath or possibly the solar wind [Desch, 1994; Kaiser, 1998; Menietti et al., 2001]. As mentioned above, the periods of the QP bursts observed by Galileo are often much more chaotic than the Ulysses results. In Figure 2, the QP emissions range from an approximate $10 \mathrm{~min}$ period at the start of the spectrogram to an approximate $40 \mathrm{~min}$ period starting at about 1930 spacecraft event time (SCET). Furthermore, from about 1600 to 1900 SCET, there are many bursts which show no obvious periodicity.

[7] Kaiser et al. [2001] presented an initial examination of the QP bursts detected by Cassini. It was suggested that since Cassini and Ulysses followed similar trajectories as they approached Jupiter, the properties of the QP bursts detected by Cassini might be similar to the Ulysses results, and the differences observed by the Galileo spacecraft may be due to the orbit of Galileo. Furthermore, although the outbound part of the trajectories of Cassini and Ulysses were different, there was a brief interval where the Cassini and Ulysses outbound trajectories were in a similar local time zone, allowing further comparison of the Ulysses, Galileo, and Cassini observations. However, Kaiser et al. [2001] found that the periods observed by Cassini of the QP bursts were rather chaotic and more similar to the Galileo results than the Ulysses observations, both on the inbound and outbound part of its trajectory. The simple QP15 inbound and QP40 outbound picture of Ulysses [MacDowall et al., 1993] is not observed in the Cassini data. Inbound, the periodicity often ranged from $<2$ min to a few tens of minutes. Furthermore, it is often difficult in the Cassini observations to determine the periodicity of the bursts, since it appears that many short period bursts occur on top of each other or that the emission appears to occur randomly. On the outbound portion of the Cassini trajectory, 40 min periodicities were occasionally observed, but many other periods were also detected, with most of the events containing many shorter-period bursts. Although a detailed survey was not performed, Kaiser et al. [2001] suggested that a histogram of the period of the Cassini observations of QP bursts would probably be much broader than the Ulysses results [MacDowall et al., 1993], with a large peak at 1 to $2 \mathrm{~min}$. It should be noted that Ulysses would not have detected the shorter-period bursts detected by Cassini and Galileo due to the $144 \mathrm{~s}$ sampling rate of the Ulysses observations. However, this does not explain the differences between the Ulysses and the Galileo/Cassini results for the larger periods ( $>5 \mathrm{~min})$. Kaiser et al. [2001] suggested that perhaps the emission mechanism had changed since the 1991/1992 Ulysses flyby of Jupiter. It is also possible that the "disappearance" of strongly periodic QP bursts could be due to multiple QP burst sources being visible to Cassini and Galileo. Simultaneous observations of multiple sources would produce a more confused picture of the periodicity of the bursts. Ulysses has the opportunity to reexamine the characteristic of QP bursts and explore these possibilities during the Ulysses distant $(\sim 1 \mathrm{AU})$ flyby of Jupiter in 2003-2004.

[8] This paper will examine the joint observations of QP bursts by the Galileo and Cassini spacecraft that were obtained during the Cassini gravity assist flyby of Jupiter.
The characteristics and modes of the Cassini and Galileo instruments will be discussed in the next section. The three types of joint observations will then be discuss in detail, and the characteristics of the QP bursts observed by each spacecraft will be examined. Preliminary Cassini-Radio and Plasma Wave Science (RPWS) direction-finding results will also be presented. A discussion of these results and a comparison with other periodic events in the Jovian system (energetic particles, X-ray auroral hot spots, and auroral UV emissions) will follow and include an examination of two possible source regions of the QP bursts.

\section{Instrument Description}

[9] The RPWS consists of five receivers, a Langmuir Probe, three electric antennas, and a triaxial search coil magnetometer (see Gurnett et al. [2004] for a detailed description of the instrument). The receivers cover a range from $\sim 1 \mathrm{~Hz}$ to $16 \mathrm{MHz}$ for electric fields and $\sim 1 \mathrm{~Hz}$ to $12 \mathrm{kHz}$ for magnetic fields. This study primarily uses the high-frequency receiver (HFR) which covers a frequency range of $3.5 \mathrm{kHz}$ to $16 \mathrm{MHz}$. Although a variety of instrument modes were used during the flyby, the primary mode of operation for the RPWS instrument obtained a complete frequency sweep from $3.5 \mathrm{kHz}$ to $16 \mathrm{MHz}$ every $25.25 \mathrm{~s}$, with a $25 \mathrm{kHz}$ wide measurement band stepped in $50 \mathrm{kHz}$ increments from $325 \mathrm{kHz}$ to $4025 \mathrm{kHz}$, and a $25 \mathrm{kHz}$ wide measurement band stepped in $200 \mathrm{kHz}$ increments from $4.025 \mathrm{MHz}$ to $16.025 \mathrm{MHz}$. The HFR connects to the electric antenna, either a dipole with a tip-to-tip length of $18.52 \mathrm{~m}$ or a monopole antenna with a length of $10 \mathrm{~m}$. The RPWS instrument obtained the data examined in this study from 4 September 2000 to 3 October 2002 and ranged from about $1535 R_{J}$ (4 September 2000), to a closest approach distance of about $137 R_{J}$ (30 December 2000), to an outbound distance of about $8735 R_{J}$ (3 October 2002). The magnetic latitude of Cassini ranged from about $-8^{\circ}$ to about $+13^{\circ}$ before closest approach and from about $-13^{\circ}$ to about $+6^{\circ}$ after closest approach.

[10] The Galileo Plasma Wave System (PWS) consists of three sweep frequency receivers, a wideband receiver, an electric dipole antenna, and two search coil magnetometers. The three sweep frequency receivers cover a range from a few Hertz to 5.6 MHz. For this study the medium-frequency sweep frequency receiver was used, which covers a range from $42.1 \mathrm{~Hz}$ to $160 \mathrm{kHz}$ in 112 logarithmically spaced channels. The receiver is attached to the electric dipole antenna with an effective length of $3.5 \mathrm{~m}$. The electric antenna is mounted perpendicular to the spacecraft spin axis. The receiver takes $18.67 \mathrm{~s}$ to complete a sweep of the electric field measurements and has a frequency resolution of $\sim 10 \%$ [Gurnett et al., 1992]. Galileo has orbited Jupiter since December 1995 and provides a very good survey of the equatorial magnetosphere from $\sim 9$ to $\sim 140 R_{J}$. During the Cassini flyby, the PWS instrument on Galileo obtained data from DOY 300, 2000 to DOY 037, 2001. The magnetic latitude of Galileo ranged from about $-13^{\circ}$ to $+10^{\circ}$ during this interval.

\section{Observations}

[11] Cassini and Galileo observed many episodes of quasi-periodic emissions during the Cassini flyby of Jupiter. 
We have examined the intervals when both spacecraft observed QP emissions at the same time during the roughly 3 month period (DOY 300, 2000 to DOY 037, 2001). It should be noted that there are intervals when either Galileo or Cassini observed QP bursts, but the other spacecraft did not observe any QP emissions. These single spacecraft events are usually one of three cases. The first case is when Galileo was in the magnetosphere and observed QP bursts as enhancements in the trapped continuum and Cassini was in the solar wind and observed no QP activity. This may be a sensitivity issue with Cassini, where weaker QP bursts can be detected by Galileo which is closer to the source of the emission. It is also likely in some of these cases that regions of higher density between Cassini and the source (including the magnetosheath) block the emission from reaching Cassini. This is supported by the fact that Galileo often observes the QP bursts at lower frequencies than the local plasma frequency of the solar wind at Cassini. The second case is when both spacecraft were in the solar wind and Cassini observed QP activity but Galileo did not. In this case, it is believed that it is primarily a sensitivity issue with Galileo, which is not as sensitive as Cassini in the few $\mathrm{kHz}$ to tens of $\mathrm{kHz}$ frequency range. The third case is when Galileo is near Jupiter (within about $30 R_{J}$ ). As Galileo approaches Jupiter, the local plasma density increases and the trapped continuum is not observed, resulting in no QP emissions being detected by Galileo. It is also likely that the higher-density plasma in the Io torus blocks the higherfrequency component of the QP bursts from reaching Galileo as Galileo approaches the planet (assuming a source of the QP bursts near the Jovian magnetic poles).

[12] The intervals of joint observations of the QP bursts with Galileo and Cassini also fall into three types. The first type are observations of QP bursts when both Galileo and Cassini are in the solar wind. The second type are observations when Galileo is in the Jovian magnetosphere and Cassini is in the solar wind. The third type is when both Cassini and Galileo are in the magnetosphere and both spacecraft observe quasi-periodic enhancements in the trapped continuum.

\subsection{Type 1: Both Spacecrafts in the Solar Wind}

[13] The first type of simultaneous observations of QP bursts are when both spacecrafts are in the solar wind. Figure 3 shows a frequency-time spectrogram of this type. The time interval is from 1400 to 2400 SCET on day 313 , 2000 for Cassini (top panel) and Galileo (bottom panel). Cassini is in the solar wind about $690 R_{J}$ upstream from Jupiter and Galileo is about $231 R_{J}$ from Jupiter, in the dusk region, probably in the solar wind, near the Jovian bowshock. Figure 4 shows the trajectory of Cassini and Galileo during the flyby, with the circles showing the location of Cassini and squares showing the location of Galileo in the equatorial $x-y$ plane for the intervals of simultaneous observations of QP emissions that are discussed in this paper. Figure 3 shows that both spacecraft observe a wide range of radio and plasma wave emissions during this interval. Some of the emissions are observed at both spacecraft at approximately the same time, for example, the four solar type III radio bursts (most easily seen in the Cassini data at approximately 1500, 2020, 2330, and 2340 SCET), while other emissions are observed only by one

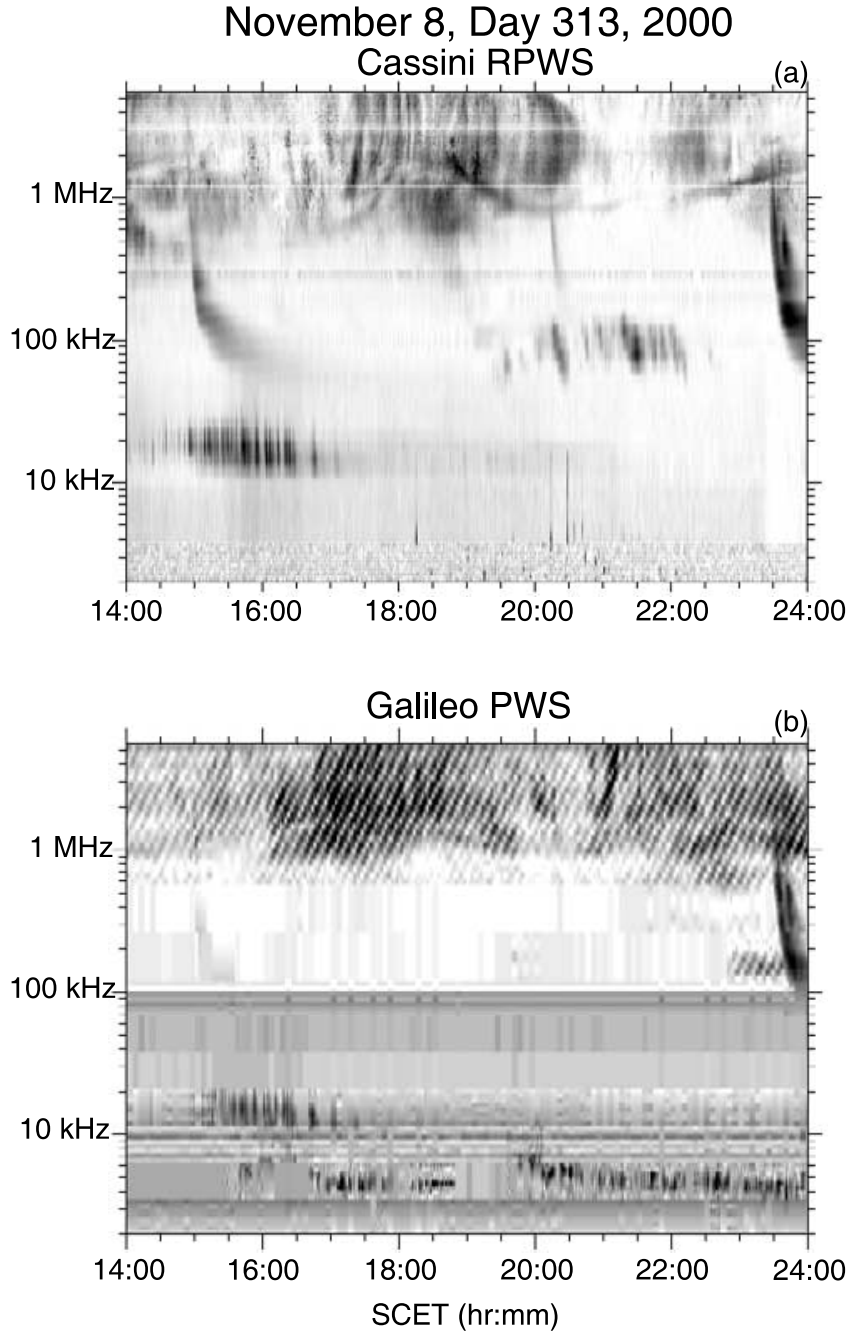

Figure 3. Frequency-time spectrograms showing Jovian radio emissions detected by Cassini (top panel) and Galileo (bottom panel) on 8 November 2000, during a period when both spacecraft were in the solar wind. QP bursts are detected by both spacecraft from about 1430 to 1700 SCET. The QP bursts detected by Cassini ranged from about $10 \mathrm{kHz}$ to $30 \mathrm{kHz}$, and the Galileo emissions ranged from about $10 \mathrm{kHz}$ to $20 \mathrm{kHz}$. Cassini is located about $690 R_{J}$ from Jupiter at 10.8 hours LT, and Galileo is about $231 R_{J}$ from Jupiter at 18.9 hours LT.

spacecraft (Langmuir waves observed by Galileo at about 4 to $6 \mathrm{kHz}$, the broadband kilometric (bKOM) emissions observed at about $100 \mathrm{kHz}$ by Cassini (1930 to 2230 SCET) and the narrowband kilometric (nKOM) observed by Galileo at about $150 \mathrm{kHz}$ at 2300 SCET. The differences in the characteristics of the emissions and the types of emissions detected by the spacecraft observed at their respective locations can provide important information about the beaming patterns and source characteristics of those emissions (for example, Kaiser et al. [2000] used radio wave data obtained from the Cassini and Wind spacecraft to investigate the beaming characteristics of the Jovian decameter (DAM) and hectometer (HOM) wavelength emissions). 


\section{Position of Cassini and Galileo}

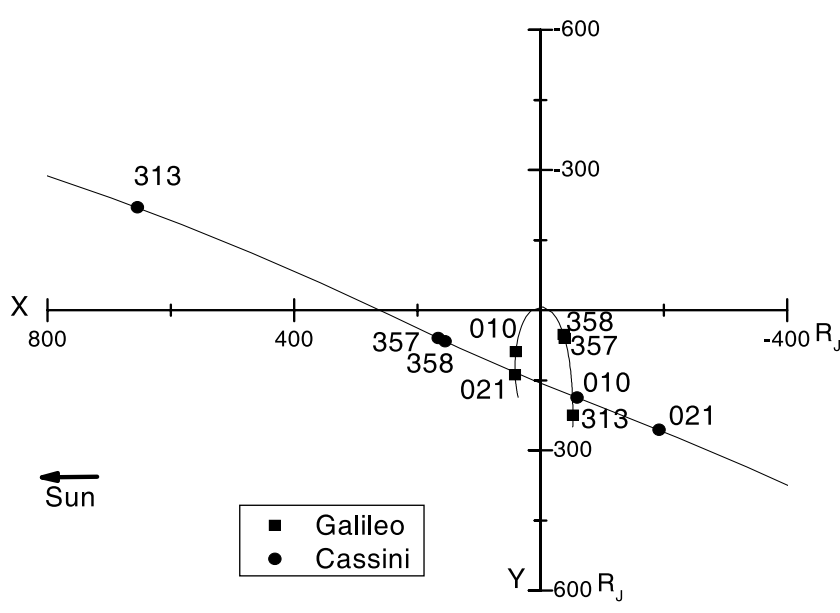

Figure 4. The trajectory of Cassini and Galileo during the flyby period, with the circles showing the location of Cassini and squares showing the location of Galileo in the equatorial $x-y$ plane for the periods of simultaneous observations of QP emissions that are discussed in this paper.

[14] Another example of an emission that is observed at both spacecraft at roughly the same time is a burst of quasiperiodic radio emission detected from about 1430 to 1700 SCET. The QP bursts detected by Cassini ranged from about $10 \mathrm{kHz}$ to $30 \mathrm{kHz}$, and the Galileo emissions ranged from about $10 \mathrm{kHz}$ to $20 \mathrm{kHz}$. This difference in the observed upper frequency range is probably due to a sensitivity difference between the two instruments in this frequency range. The two spacecraft are at very different local times during this interval, with Cassini at 10.8 hours LT and Galileo at 18.9 hours LT (see Figure 4). The magnetic latitude of the two spacecraft ranged from about $0^{\circ}$ to $-5.9^{\circ}$ for Cassini and from about $8.6^{\circ}$ to $0^{\circ}$ for Galileo during this event.

[15] Figure 5 shows the integrated power of the QP emissions shown in Figure 3 for the interval from 1500 to $1715 \mathrm{SCET}$ in a band from 15 to $20 \mathrm{kHz}$. The data from both spacecraft was averaged over $1 \mathrm{~min}$, and the Cassini integrated power results were shifted downward in amplitude to more easily compare the two data sets. The Cassini data was also shifted "earlier" by 2 min (Cassini data obtained at 1500 SCET is plotted at 1458 SCET). The reason for this shift will be discussed below. The QP emissions detected by both spacecraft have very similar periods (roughly 10 min periods from 1500 to 1615 SCET and $\sim 20$ min periods from 1615 to 1715 SCET). As reported by Hospodarsky et al. [1998] and Kaiser et al. [2001], the QP bursts detected by Galileo and Cassini often appear to contain "subbursts" with periods of less than a few minutes (very similar to the "subbursts" detected by Voyager [Kurth et al., 1989]). Figure 5 also shows that the peaks in the integrated power observed by both spacecraft are very similar during this event. A cross-correlation was performed between the nonshifted, 1 min averaged data sets, and the best correlation ( 0.88 correlation) was found when the Cassini data had a 2 min lag (for this reason, the Cassini data has been plotted in Figure 5 with the 2 min shift). This shift is very close to the difference (within the resolution of the $1 \mathrm{~min}$ averaged data and the time resolution $(\sim 20 \mathrm{~s})$ of both instruments) in the light-time distance from Jupiter to each of the spacecraft (approximately $109 \mathrm{~s}$ for this interval). To examine small differences in the observed timing of the QP radio burst, the individual channel data was examined from each instrument. Figure 6 shows the spectral density results for the $15.1 \mathrm{kHz}$ channel on Galileo and the $14.7 \mathrm{kHz}$ channel on Cassini. Again, the Cassini data was shifted downward in amplitude to more easily compare the data and the Cassini data was also shifted by the speed of light travel time difference between Jupiter and the two spacecraft (109 s). Again, it is easy to see the very good correlation between the two data sets, even though the two spacecraft are separated by $\sim 8$ hours of local time. If the emission rotated with the planet, we would expect an offset of about 4 hours, with Cassini observing the QP bursts first, followed by Galileo. As can be seen in Figures 3, 5, and 6, this shift of about 4 hours is not observed.

[16] Figure 7 shows a second example of a joint observation from 0400 to 1200 SCET on day 021,2001 for Cassini (top panel) and Galileo (bottom panel), when both spacecraft are in the solar wind. In this example, the frequency range of the frequency-time spectrograms have been reduced to emphasize the QP emissions. Cassini was about $320 R_{J}$ from Jupiter and moving down the dusk flank, while Galileo was about $144 R_{J}$ from Jupiter and upstream of Cassini (see Figure 4). A series of QP bursts are observed from about 0700 to 1000 SCET by both spacecraft and range in

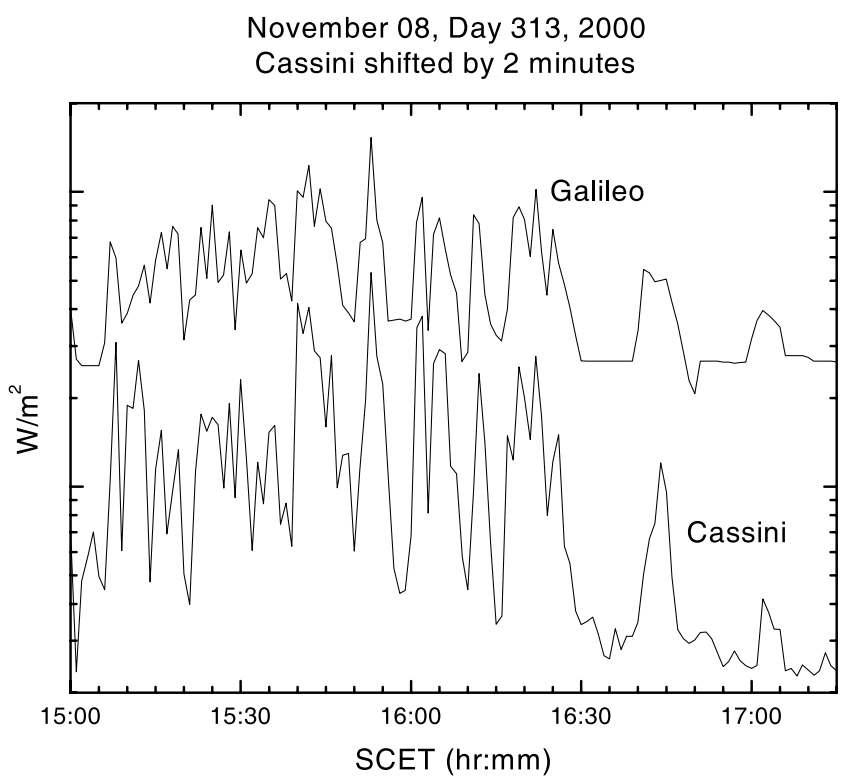

Figure 5. A plot of the integrated power flux from 15 to $20 \mathrm{kHz}$ ( 1 min averages) of the QP emissions as measured by Galileo (top curve) and Cassini (bottom curve). The Cassini data has been shifted downward to allow a more easy comparison of the data and "earlier" (to the left) by 2 min to account for the difference in the light time from Jupiter to each of the spacecraft (actual difference is $109 \mathrm{~s}$, or $1.82 \mathrm{~min}$ ). As can be seen, the QP bursts observed by the two spacecraft are very similar. 


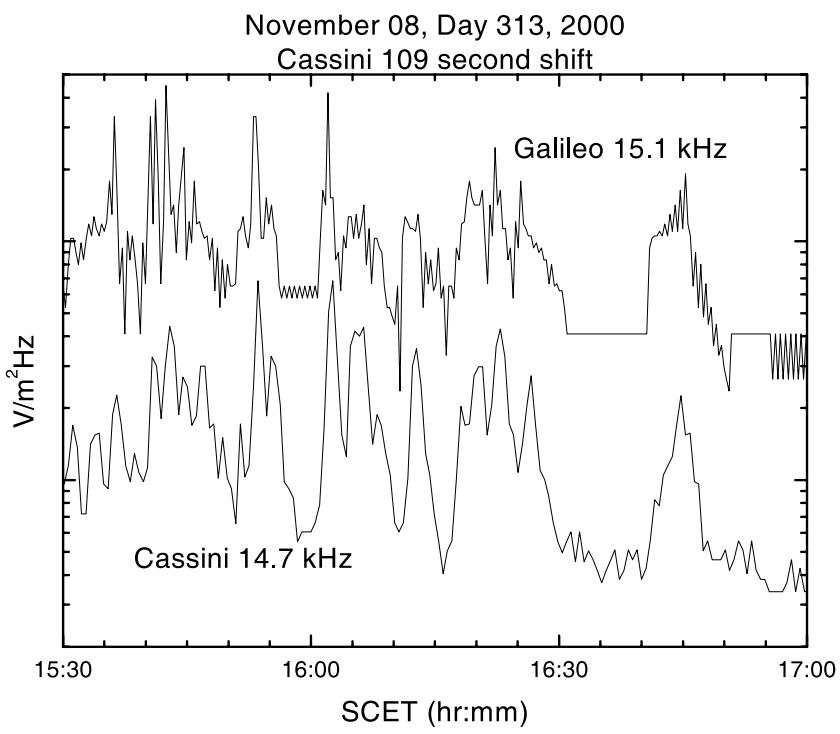

Figure 6. A plot of the Galileo $15.1 \mathrm{kHz}$ channel (top curve) and the Cassini $14.7 \mathrm{kHz}$ channel (bottom curve) for the period from 1530 to 1700 on day 313, 2000. As can be seen, both spacecraft detect the QP emissions with very similar periods and structure. The Cassini data has been shifted downward to more easily compare the two curves and "earlier" by $109 \mathrm{~s}$, which is the difference in the light time from Jupiter to each of the spacecraft.

frequency from about $5 \mathrm{kHz}$ to $15 \mathrm{kHz}$. The QP bursts in this interval are observed on top of a continuum-like emission that occurs in the same frequency range as the QP bursts. This continuum like emission is most likely Jovian Anomalous Continuum [Kaiser et al., 1992; Kaiser, 1998] and is often detected with the QP bursts [Kaiser, 1998]. See Kaiser et al. [2004] for recent observations of Jovian Anomalous Continuum by Ulysses, Galileo, and Cassini.
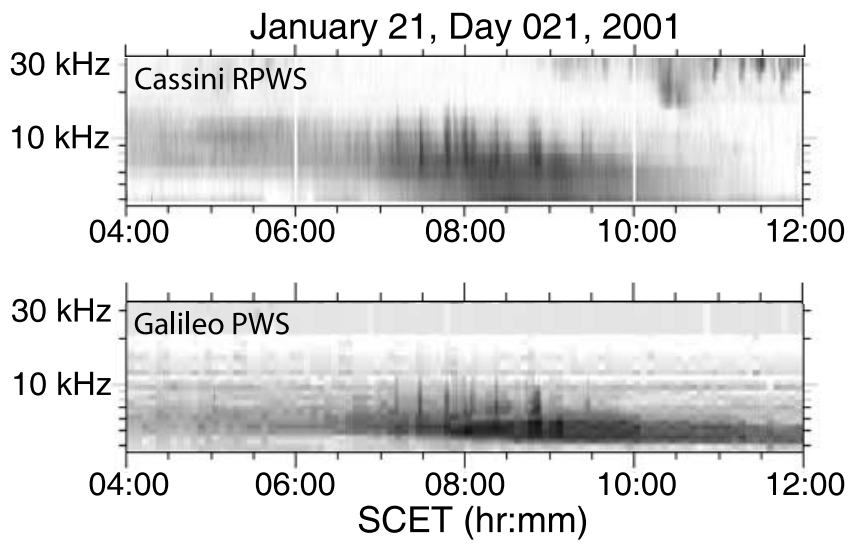

Figure 7. Frequency-time spectrograms showing QP bursts detected by Cassini (top panel) and Galileo (bottom panel) from 21 January 2001, when both spacecraft are in the solar wind. QP bursts are detected by both spacecraft from about 0700 to 1000 SCET. The QP bursts detected by both spacecraft range in frequency from about $5 \mathrm{kHz}$ to $15 \mathrm{kHz}$. Cassini is located about $320 R_{J}$ from Jupiter and at 20.5 hours LT, while Galileo was about $144 R_{J}$ from Jupiter and at 16.9 hours LT.

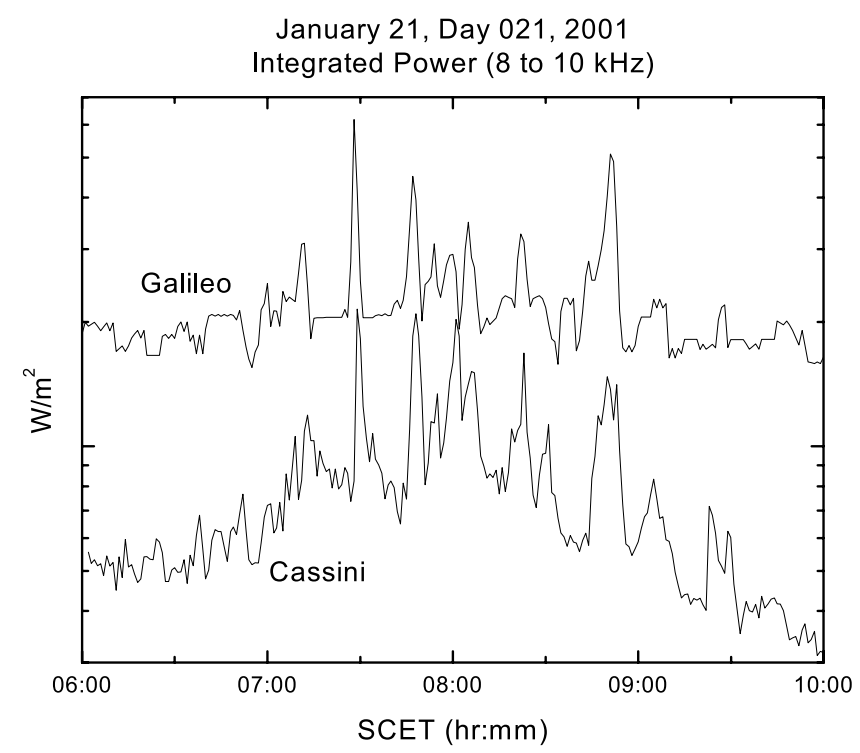

Figure 8. A plot of the integrated power flux from 8 to $10 \mathrm{kHz}$ ( 1 min averages) of the QP emissions as measured by Galileo (top curve) and Cassini (bottom curve) for 21 January 2001. The Cassini data has been shifted downward to allow a more easy comparison of the data. In this example, the Cassini data has not been shifted "earlier" (to the left) to account for the difference in the light time from Jupiter to each of the spacecraft (actual difference is $\sim 42 \mathrm{~s}$, or $0.7 \mathrm{~min}$ ). As can be seen, the QP bursts observed by the two spacecraft are very similar, though the Cassini peaks occur slightly after the Galileo peaks.

[17] Figure 8 shows the integrated power from 8 to $10 \mathrm{kHz}$ from both spacecraft for the interval from 0600 to 1000 SCET, again with the Cassini amplitude shifted downward to more easily compare the two data sets. As can be seen, the QP bursts observed by both spacecraft match very well, especially when comparing the occurrence of the peaks in the emissions. The Cassini data was not shifted in time for Figure 8, and a small lag between the Cassini peaks compared with the Galileo peaks can be observed (the Cassini peaks occurs slightly later in time than the Galileo peaks, as expected if the source region is closer to Galileo). Figure 9 shows the individual channel data for $8.25 \mathrm{kHz}$ channel from Galileo and the $8.4 \mathrm{kHz}$ channel from Cassini. The Cassini data are shifted by $42 \mathrm{~s}$ in this figure, which is the light-time difference between Jupiter and each spacecraft. Again, it is easy to see the very good agreement between the two spacecraft. All the large peaks in the Galileo data have a corresponding peak in the Cassini data that occur at nearly the same time (when the $42 \mathrm{~s}$ shift is applied) even though the spacecraft are separated by almost 4 hours in local time. It should be noted that the Galileo lossy compression tends to produce step-level changes for weaker emission. These step-level changes can be most easily seen during the last 50 min of Figure 9.

\subsection{Type 2: Cassini in Solar Wind, Galileo in Magnetosphere}

[18] The second type of joint observations occur when Galileo is in the Jovian magnetosphere and Cassini is in the 


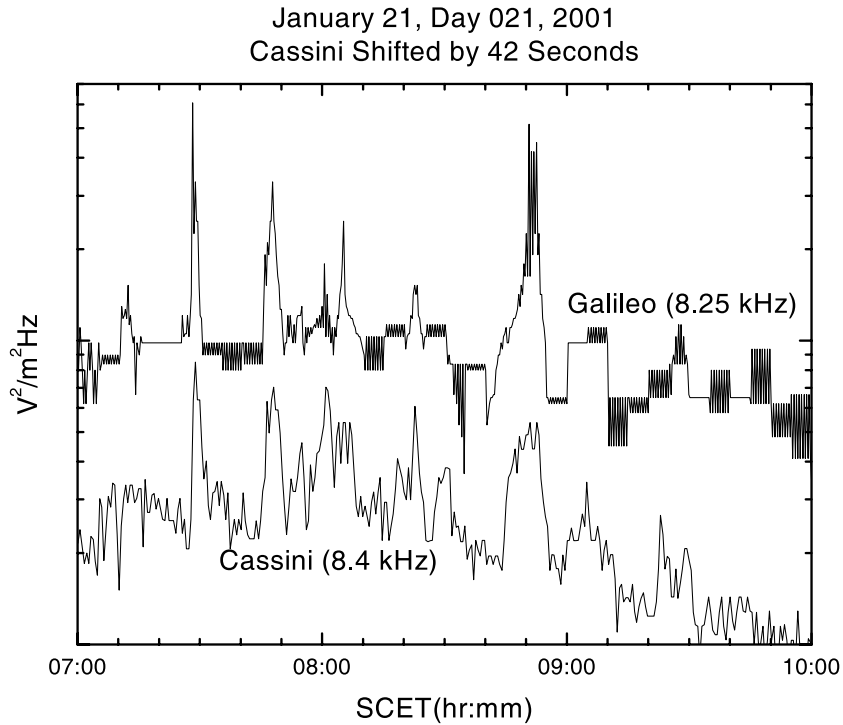

Figure 9. A plot of the Galileo $8.25 \mathrm{kHz}$ channel (top curve) and the Cassini $8.4 \mathrm{kHz}$ channel (bottom curve) for the period from 0700 to 1000 on day 021,2001 . The Cassini data has been shifted by $42 \mathrm{~s}$, the difference in the light time from Jupiter to each of the spacecraft. As can be seen, both spacecraft detect QP bursts with very similar periods and structure, and the $42 \mathrm{~s}$ shift causes the peaks in the signals from the two spacecrafts to match very well. The "stair step" like data most easily seen during the last $50 \mathrm{~min}$ of the Galileo data is produced by the Galileo lossy compression, which tends to produce step-level changes for weaker emission.

solar wind. Figure 10 shows an example of this type of observation from 0000 to 0500 SCET on 22 December 2000. Galileo (bottom panel) detects enhancements of the trapped continuum from about 1 to $20 \mathrm{kHz}$ during the same time interval that Cassini (top panel) observed periodic like emissions from about 15 to $25 \mathrm{kHz}$. Cassini is approximately $176 R_{J}$ upstream from Jupiter (13.3 hours LT) and Galileo is a little over $70 R_{J}$ from Jupiter and in the Jovian magnetosphere (20.2 hours LT) during this interval (see Figure 4). Again, the QP bursts detected by both spacecraft appear to be more random and have a shorter periodicity than the Ulysses observations. It is possible that these events are made up of many QP bursts that occur on top of each other, which may produce the more random periodicities that are observed. Figure 11 shows the data for the $15.1 \mathrm{kHz}$ channel from Galileo and the $17.8 \mathrm{kHz}$ channel from Cassini. The Cassini data have been shifted downward in amplitude and shifted by $25 \mathrm{~s}$ in time, which is the lighttime difference between Jupiter and each spacecraft. It is easy to see the very good agreement in the QP emissions detected by the two spacecraft, even the shorter-period "subbursts" are usually detected by both spacecraft simultaneously. Figure 12 shows the $1 \mathrm{~min}$ averages of the integrated power from 10 to $20 \mathrm{kHz}$ (Galileo) and 15 to $20 \mathrm{kHz}$ (Cassini). The Cassini data has been shifted downward in amplitude to more easily compare the two data sets. Again, the emissions detected by both spacecraft are very similar. A cross correlation analysis was performed on the 1-min averaged data from Figure 12, resulting in a broader peak in the correlation at a value of no lag and a $1 \mathrm{~min}$ lag in the Cassini data, which agrees very well with the light time difference of $25 \mathrm{~s}$.

[19] Figure 13 shows an example from the next day (23 December, Day 358, 2000). Again, Cassini is in the solar wind and Galileo is in the Jovian magnetosphere. The QP bursts detected by Cassini during this event appear to consist of a series of bursts with a period of about $30 \mathrm{~min}$, with many bursts with shorter periods "on top of" the 30 min QP bursts. It is more difficult to see the QP bursts in the Galileo data shown in Figure 13. However, if the integrated power is examined (see Figure 14), the QP bursts are much easier to see for both spacecraft. Figure 14 shows the integrated power from 0645 to 1045 SCET for Galileo
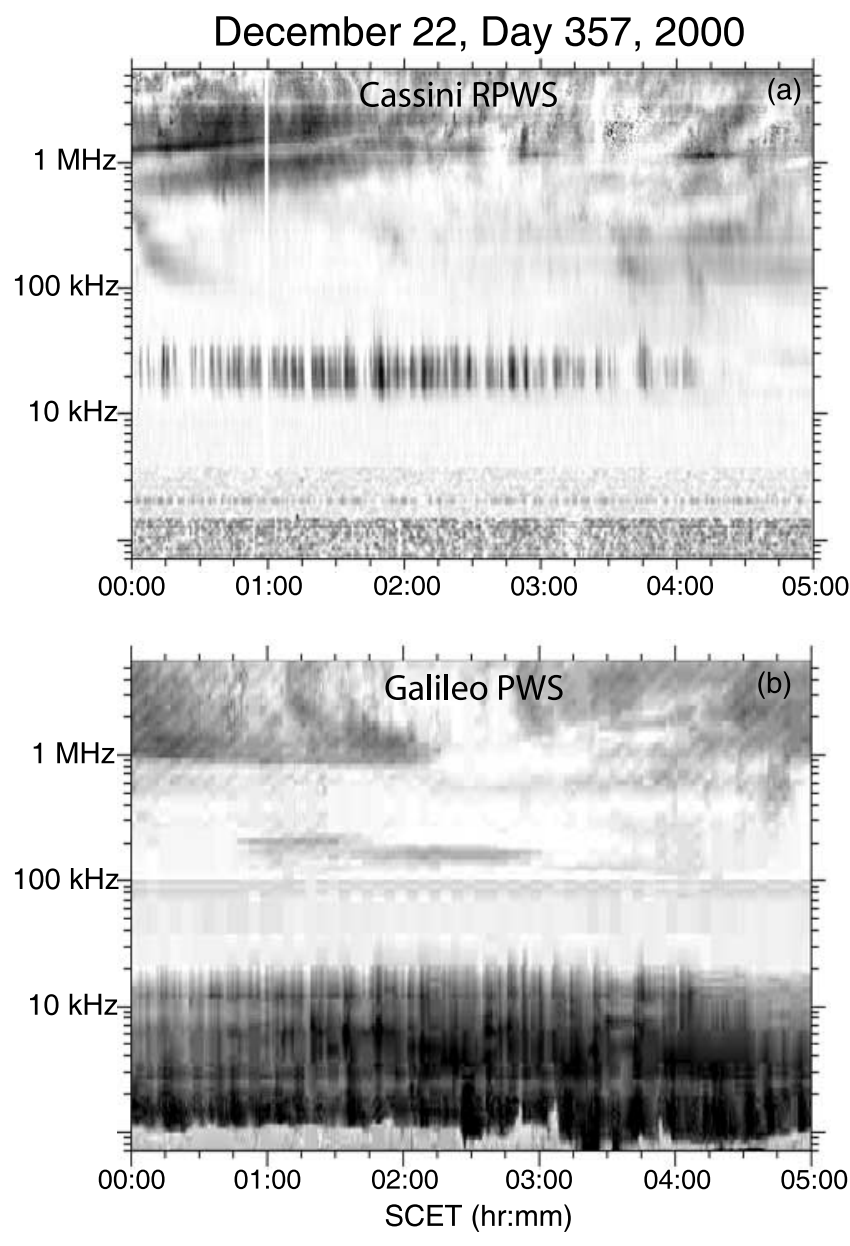

Figure 10. Frequency-time spectrograms showing QP bursts detected by Cassini (top panel) and Galileo (bottom panel) from 22 December 2000, when Cassini was in the solar wind and Galileo was in the Jovian magnetosphere. QP bursts are detected by both spacecraft from about 0000 to 0415 SCET, with Galileo continuing to observe periodic enhancement in the trapped continuum 0500 SCET. Galileo detected enhancements of the trapped continuum from about 1 to $20 \mathrm{kHz}$ during the same period and Cassini observed periodic-like emissions from about 15 to $25 \mathrm{kHz}$. Cassini was about $176 R_{J}$ from Jupiter and at 13.3 hours LT, while Galileo was about $71 R_{J}$ from Jupiter and at 20.2 hours LT. 


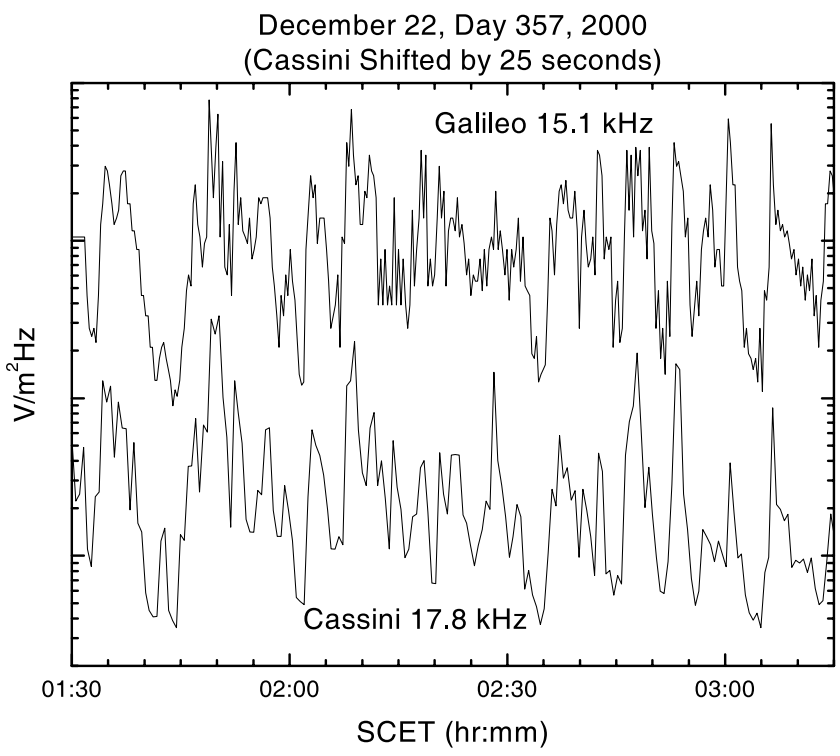

Figure 11. A plot of the Galileo $15.1 \mathrm{kHz}$ channel (top curve) and the Cassini $17.8 \mathrm{kHz}$ channel (bottom curve) for the period from 0130 to 0315 on day 357, 2000. The Cassini data has been shifted by $25 \mathrm{~s}$, the difference in the light time from Jupiter to each of the spacecraft. As can be seen, both spacecraft detect QP bursts with very similar periods and structure, even though Galileo is in the magnetosphere during this event.

from 6 to $10 \mathrm{kHz}$ and for Cassini from 7 to $15 \mathrm{kHz}$. Again, Cassini is shifted downward to more easily compare the two data sets. A Fourier transform (not shown) of the data shown in Figure 14 shows peaks in the period of the QP

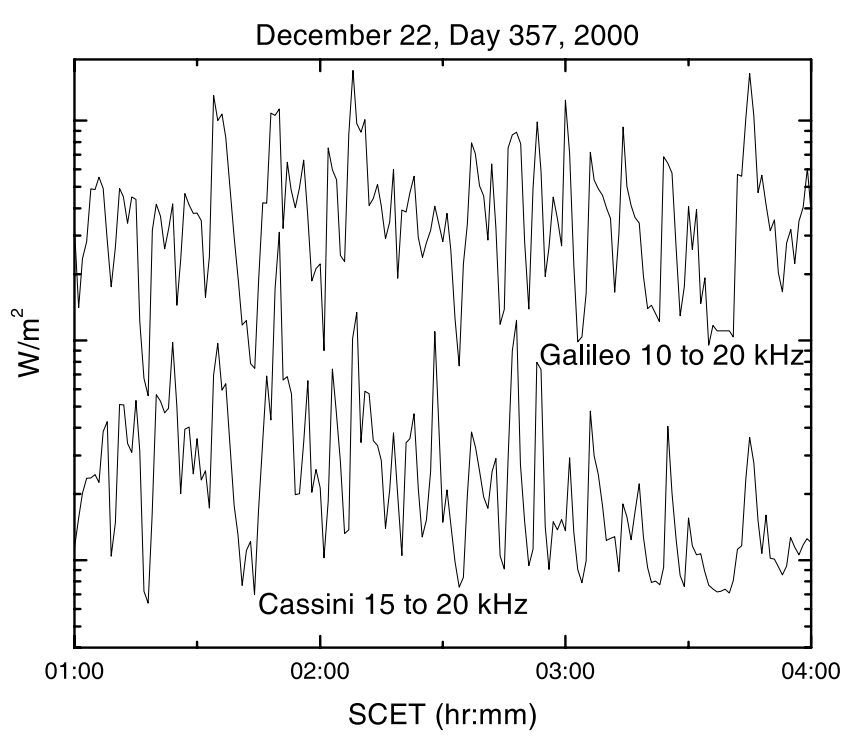

Figure 12. A plot of the integrated power flux from 10 to $20 \mathrm{kHz}$ (1 min averages) of the QP emissions as measured by Galileo (top curve) and from 15 to $20 \mathrm{kHz}$ by Cassini (bottom curve) for 22 December 2000. The Cassini data has been shifted downward to allow a more easy comparison of the data. The QP bursts observed by the two spacecraft are very similar, with much of the fine structure of the QP bursts appearing at both spacecraft.
December 23, Day 358, 2000 Cassini RPWS

(a)
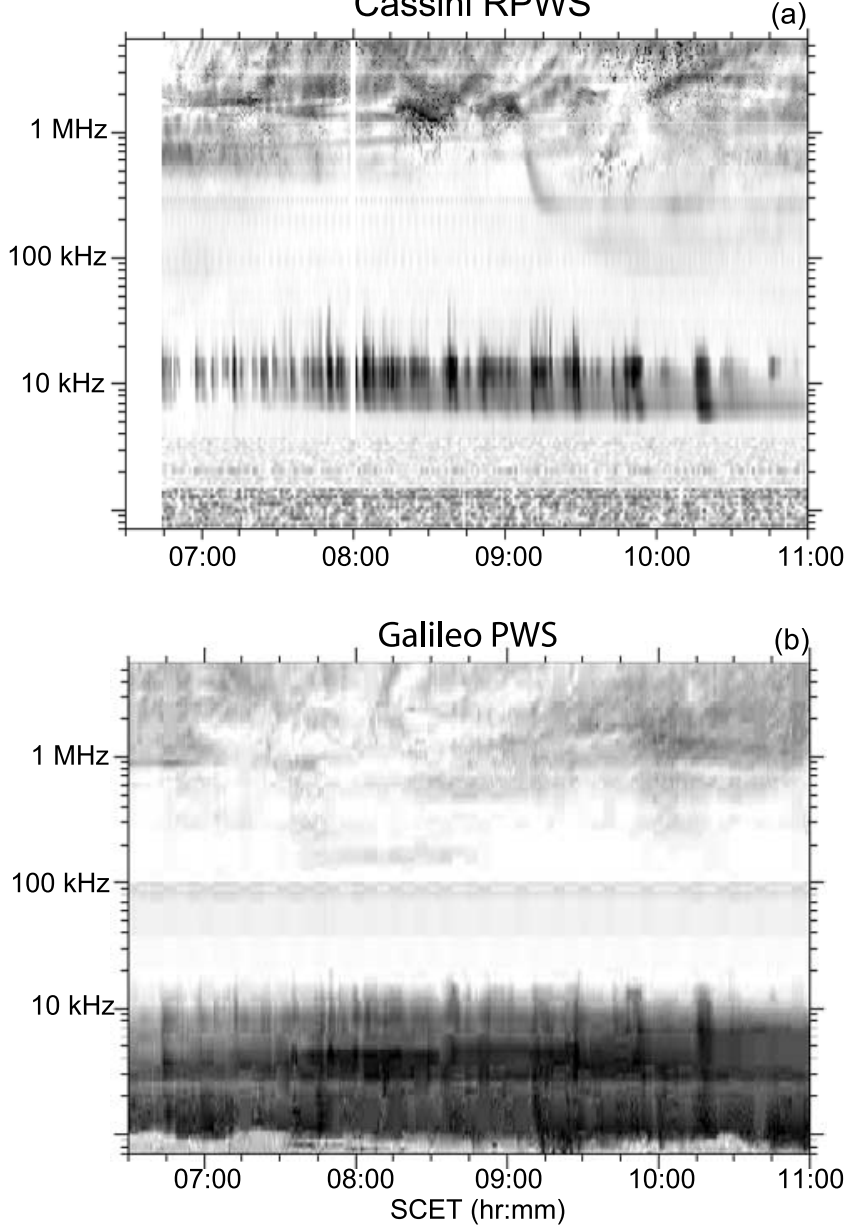

Figure 13. Frequency-time spectrograms showing QP bursts detected by Cassini (top panel) and Galileo (bottom panel) from 23 December 2000, when Cassini was in the solar wind and Galileo was in the Jovian magnetosphere. QP bursts are detected by both spacecraft from about 0645 to 1100 SCET. Galileo detected enhancements of the trapped continuum from about 1 to $15 \mathrm{kHz}$ during the same period and Cassini observed periodic-like emissions from about 5 to $40 \mathrm{kHz}$. Cassini was located about $166 R_{J}$ from Jupiter and at 13.7 hours LT, while Galileo was located about $62 R_{J}$ from Jupiter and at 2.4 hours LT.

bursts at $\sim 12$ and $\sim 18$ min. Very good correlation between the peaks of the emissions are seen between the two spacecraft, especially from about 0830 to 1030 SCET. The light time difference between the two spacecraft is $25 \mathrm{~s}$ during this event. A cross correlation was run on the data from Figure 14, resulting in a maximum at no shift (with a smaller peak at a $1 \mathrm{~min}$ lag) of the Cassini data.

[20] As can be seen from Figures 10 and 13, during the intervals that Cassini is in the solar wind and Galileo is in the magnetosphere, Galileo observes the QP bursts at much lower frequencies. This difference comes from the fact that the lower-frequency component of the emission cannot propagate across the magnetopause into the higher density magnetosheath (and into the solar wind). Zarka et al. [2004] has examined 6 months of the Cassini-RPWS measurements during the Jupiter flyby and have found that the QP bursts 


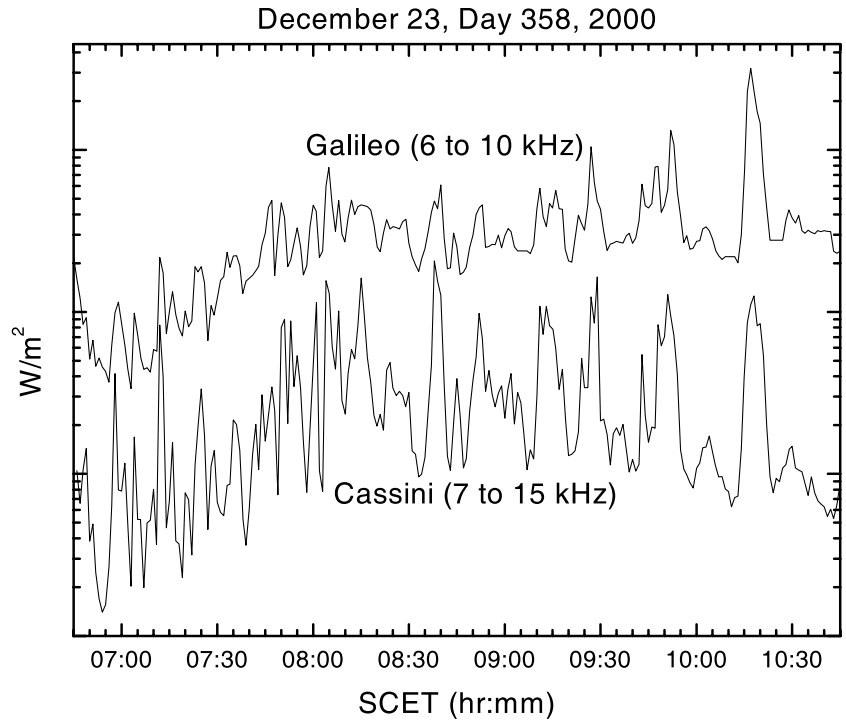

Figure 14. A plot of the integrated power flux from 6 to $10 \mathrm{kHz}$ (1 min averages) of the QP emissions as measured by Galileo (top curve) and from 7 to $15 \mathrm{kHz}$ by Cassini (bottom curve) for 23 December 2000. The Cassini data has been shifted downward to allow a more easy comparison of the data. The QP bursts observed by the two spacecraft are very similar, with much of the fine structure of the $\mathrm{QP}$ bursts appearing at both spacecraft.

spectrum quasi-steadily increases down to $3.5 \mathrm{kHz}$. Power estimates of the QP bursts detected are also given by Zarka et al. [2004] in Table 2.

\subsection{Type 3: Both Spacecraft are in the Magnetosphere}

[21] The last example occurred when both Galileo and Cassini were in the magnetosphere (see Kurth et al. [2002] for a discussion of this interval of both spacecraft crossing the magnetopause within tens of minutes of each other (or more likely, the magnetopause crossing over the two spacecraft)). Figure 15 shows the frequency-time spectrogram from Cassini (top panel) and Galileo (bottom panel) from 10 January 2001. The data have been plotted in color to indicate amplitude (red is most intense, blue is least intense) instead of a gray scale to more easily show the enhancements in the trapped continuum detected by both spacecraft. When the spacecraft crosses from the magnetosphere into the magnetosheath, the lower-frequency cutoff of the trapped continuum jumps in frequency since the trapped continuum cannot propagate across the magnetopause into the higherdensity magnetosheath. During this interval, Cassini encounters a range of densities, which causes the low-frequency cutoff of the trapped continuum to vary a great deal, making it difficult at times to observe the quasi-periodic enhancements in the continuum. Arrows have been added to help point out some of the stronger enhancements in the continuum that are detected by both spacecraft. As can be seen, there is a very good agreement between the enhancements in the continuum detected by Galileo and the enhancements detected by Cassini. During this time interval, Cassini is about $200 R_{J}$ from Jupiter and located at about 19.3 hours $\mathrm{LT}$, and Galileo is about $100 R_{J}$ from Jupiter and located at about 16.4 hours LT. The distance between the two spacecraft at this time is about $145 R_{J}$.

[22] Figure 16 shows the preliminary results of direction finding analysis performed on the RPWS data from DOY 357, at about 0135 SCET (from the group of QP bursts shown in Figure 10, corresponding to the intense emission visible on Figures 11 and 12 at $\sim 0135$ SCET). These results are "preliminary" in the sense that (1) only a short data sample was analyzed and (2) approximate effective length vectors of the electric dipoles representing Cassini-RPWS antennas were used, as determined by electrolytic tank measurements of a $1 / 20$ th scale model of the spacecraft (so-called "rheometric" measurements [Rucker et al., 1996]). These values will be improved in the paper by Vogl et al. [2004] on the Cassini RPWS antenna calibration but should not have any serious impact on the QP bursts apparent source location because this source is far (angularly) from Jupiter during this interval. The improved antenna calibration should change the results by no more then a few degrees. However, the improved calibrations

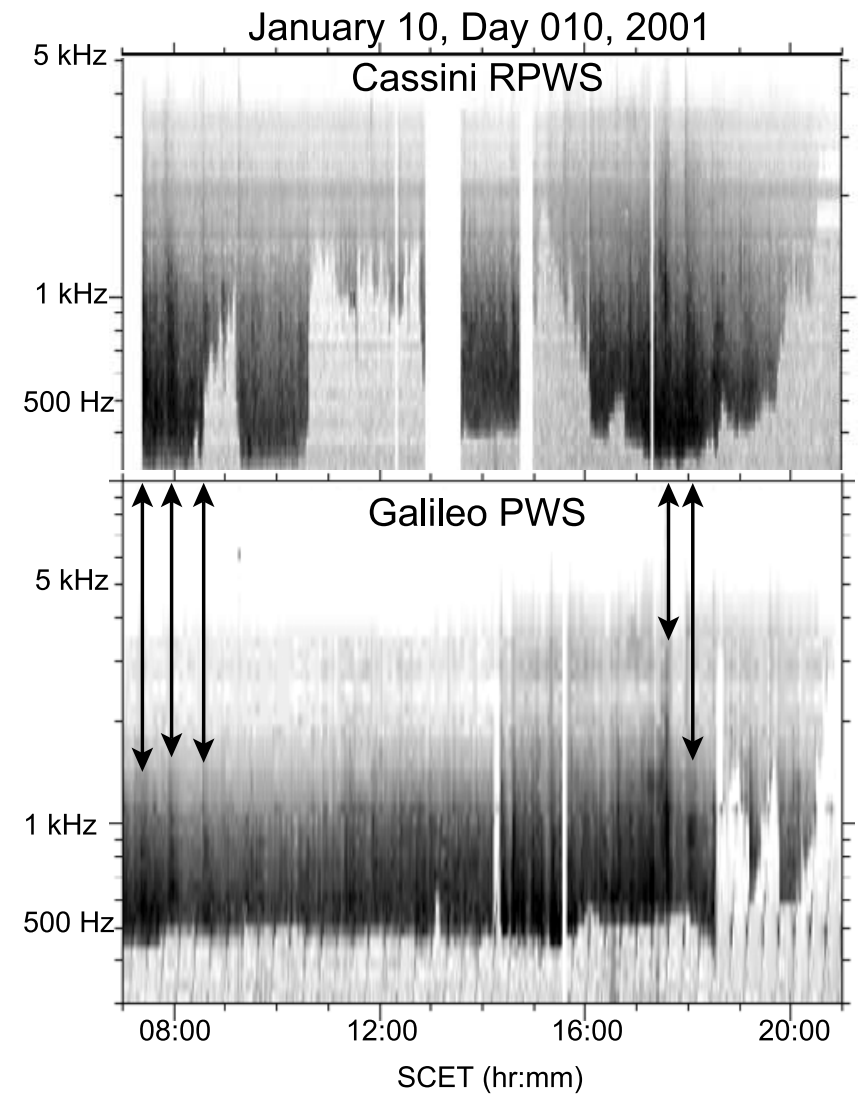

Figure 15. A frequency-time spectrogram from Cassini (top panel) and Galileo (bottom panel) from 10 January 2001, during a period when both spacecraft were located inside the Jovian magnetosphere. The data has been plotted in color to indicate amplitude with red being the most intense and blue the least intense. Arrows have been added to help point out some of the stronger enhancements in the continuum that are detected by both spacecraft. As can be seen, there is a very good agreement between the enhancements in the continuum detected by Galileo and Cassini. See color version of this figure at back of this issue. 


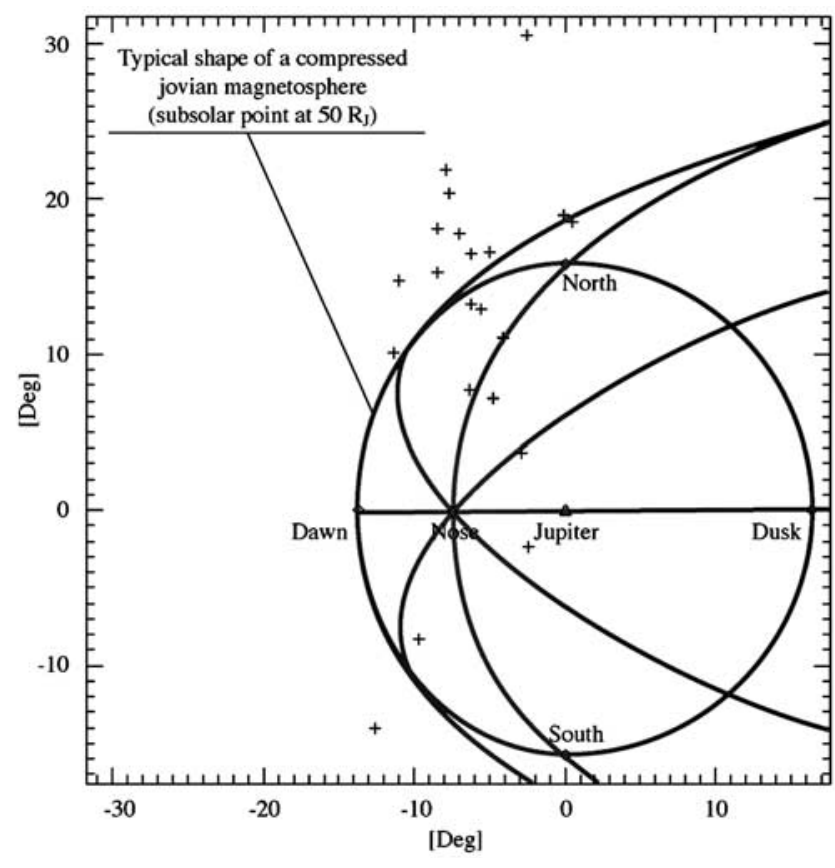

Figure 16. A plot of the apparent direction of arrival of QP bursts from DOY 357, 2002, at $\sim 20 \mathrm{kHz}$ (from the group of QP bursts shown in Figure 10, corresponding to the intense emission visible on Figures 11 and 12 at $\sim 0135$ SCET), projected (the crosses) on the plane of the sky. The axes are the azimuth and colatitude in a Jupitercentered XYZ frame, with $\mathrm{Z}$ along the Jupiter to Cassini axis, $\mathrm{Y}$ is the plane containing Jupiter's rotation axis and $\mathrm{Z}$, pointing in Jupiter's north pole direction, and X completes the right-hand triad. Superimposed is the sketch of the orientation and size of the Jovian magnetosphere as seen from Cassini in the same reference frame. The magnetosphere is seen from the dayside, slightly off the Sun-planet axis. Remarkable points (dawn and dusk terminators, north and south pole directions, and the magnetosheath nose) are represented for a compressed magnetopause with a subsolar point at $50 R_{J}$.

may modify the polarization results, so they will be discussed in a future work. Figure 16 shows the apparent direction of arrival of the waves at $\sim 20 \mathrm{kHz}$, projected (the crosses) on the plane of the sky. The axes are the azimuth and colatitude in a Jupiter-centered XYZ frame, with $\mathrm{Z}$ along the Jupiter to Cassini axis, $\mathrm{Y}$ is the plane containing Jupiter's rotation axis and Z, pointing in Jupiter's north pole direction, and $\mathrm{X}$ completes the right-hand triad. Superimposed is the sketch of the orientation and size of the Jovian magnetosphere as seen at this time from Cassini in the same reference frame. The magnetosphere is seen from the dayside, slightly off the Sun-planet axis. Remarkable points (dawn and dusk terminators, north and south pole directions, and the magnetosheath nose) are represented for a compressed magnetopause with a subsolar point at $50 R_{J}$ (of course, this is only indicative, and the magnetosphere can be twice as extended).

[23] From Figure 16, it appears that the direction of arrival of the QP emission during this interval is from the northern hemisphere, far above the equatorial plane. This apparent source region is far above the region where the local $\mathrm{f}_{\text {ce }}$ is $\sim 20 \mathrm{kHz}$ ( 5 to 7 degrees north of the equator on this figure). This suggests that even at $20 \mathrm{kHz}$ (i.e., not even in the long diffused tail), the radiation undergoes a complex propagation process before exiting the magnetosphere at high latitude. This direction-finding analysis reveals for this example the exit point of the QP bursts into the solar wind and not the actual source region. Low-frequency emissions generated near Jupiter can escape from the magnetosphere in all directions if their frequency is at least twice the plasma frequency of the solar wind at the nose of the bow shock. For an emission with a frequency below $2 \mathrm{f}_{\mathrm{psw}}$, it can only escape into the solar wind tailward of the nose, where the magnetosheath plasma frequency becomes low enough to allow the wave to propagate through. For a more detailed discussion of the interaction of the lower frequency waves and the magnetosheath, see Desch [1994], Desch and Farrell [2000], Steinberg et al. [2004], Kaiser et al. [2004], and Zarka et al. [2004].

\section{Discussion}

[24] The simultaneous observations of QP bursts by Cassini and Galileo show very similar characteristics between the emissions detected by the two spacecraft, even when the spacecraft are at very different locations. This similarity suggests that the QP emission has a "strobe light" or "flash bulb" source, which emits the radio signal over a large solid angle and is not a narrow beam rotating with the planet. The small differences in the time that each spacecraft detects the QP bursts match very well with the light travel time differences between each of the spacecraft and the planet Jupiter (within the resolution of the sampling rate of the receivers) and does not match the expected difference if the emissions rotated with the planet. The observations of the QP bursts as enhancements in the trapped continuum emissions during the intervals the spacecraft are in the Jovian magnetosphere also suggest that the emission is "beamed" over a large area and suggest that the emission source is near Jupiter. This source emits the quasi-periodic radio bursts over a wide frequency range (few hundred $\mathrm{Hz}$ to a few hundred $\mathrm{kHz}$ ). These bursts propagate though the magnetosphere and appear "on top of" the trapped continuum. At the magnetosheath, Desch [1994] showed that the propagation of the emission through the higher-density sheath can produce the characteristic spectral shape of the QP bursts detected in the solar wind (similar to solar type III's emissions, but on a much shorter time scale). The lack of dispersion in the lower-frequency part of the QP bursts detected in the magnetosphere [Menietti et al., 2001] supports the hypothesis that the source of the dispersion is in the magnetosheath. The slowing and spreading of the lowerfrequency part of the emission in the Jovian magnetosheath may also be responsible for the Jovian VLF emissions (also called the Jovian anomalous continuum) [Kaiser, 1998; Kaiser et al., 2004] and at Earth (in the Earth's magnetosheath) to explain the dispersion observed in the terrestrial LF bursts [Steinberg et al., 2004].

[25] The Jovian system appears to be rich in other periodic events, many with periods similar to the QP bursts. As discussed above, Ulysses detected $\sim 40$ min periodicities in the energetic particles [Simpson et al., 1992b; McKibben 
et al., 1993; Desch, 1994]. Some of these electron events were found to be well correlated with the QP40 bursts. However, it is not clear that the $\mathrm{MeV}$ electrons produce the corresponding radio emission [Desch, 1994]. Similar quasiperiodic bursts of electrons have been detected by the Cassini MIMI instrument (N. Krupp, private communications, 2002), but no obvious correlation between the radio bursts detected by Cassini or Galileo have been observed.

[26] Similar periodic behavior has been observed in the $\mathrm{X}$-ray spectrum of the Jovian auroral region [Gladstone et al., 2002]. Observations made by the high-resolution camera (HRC) of the Chandra X-ray Observatory on 18 December 2000 show that the northern auroral X-rays are concentrated in a "hot spot" within the main ultraviolet auroral oval at high magnetic latitudes (roughly $60^{\circ}$ to $70^{\circ}$ north latitude and $160^{\circ}$ to $180^{\circ}$ system III longitude). The light curve and power spectrum of this "hot spot" showed a very strong $\sim 45$ min oscillation in the emitted X-rays [Gladstone et al., 2002]. The radio observations of both Cassini and Galileo were examined during the period of the X-ray observations, and although weak QP bursts were detected during this interval by both spacecraft, the radio QP bursts were of a shorter period and no obvious direct correlation was found when compared to the X-ray bursts. Gladstone et al. [2002] also reported no comparable periodicities were detected in the Cassini solar wind data or in the Galileo and Cassini energetic particle measurements at the time of the Chandra observations. However, the periodicity of the X-ray bursts are similar to the earlier Ulysses energetic particle and QP radio burst observations [MacDowall et al., 1993], and the period that is sometimes observed in the $\mathrm{QP}$ radio bursts that are detected by Cassini and Galileo, suggesting that they may be related.

[27] Recent analysis of the ultraviolet observations from the Cassini Ultraviolet Imaging Spectrograph (UVIS) [Pryor et al., 2003] has found fluctuations in the auroral UV with periods similar to the QP bursts. Initial examination of one of these events has shown that the weak enhancements in the trapped continuum (QP bursts) detected by Galileo appear to be correlated with the peaks in the ultraviolet emissions [Pryor et al., 2003]. Unfortunately, Cassini did not detect any QP bursts during this interval. Further analysis and comparison of the Cassini UVIS data with the Cassini and Galileo radio data is continuing, and UV data from the Hubble Space Telescope (HST) from this same time period is also being examined for possible periodic structure.

[28] These multiple examples of quasi-periodic behavior suggest that many of these observations may be related. However, the lack of a clear correlation between many of the events suggests that it is not a simple relationship. Many of the periodic events appear to be related to auroral processes, which agrees with the earlier Ulysses directionfinding results for the QP 40 burst appearing to come from near the south Jovian polar region. The initial direction finding results with Cassini showing that the direction of arrival of the QP emission for one event is from the northern hemisphere, far above the equatorial plane (see Figure 16) do not necessarily disagree with these earlier Ulysses direction finding results showing the source region near the Jovian southern polar region (corresponding to a few degrees in Figure 16). The Ulysses direction finding results reported by MacDowall et al. [1993] were for a higherfrequency component of the QP emissions $(27 \mathrm{kHz}$ to $48 \mathrm{kHz}$ ) and were done when Ulysses was at $30^{\circ} \mathrm{S}$ latitude, near the dusk terminator and $27.5 R_{J}$. The lower-frequency part of the emissions is not expected to propagate in a straight line from the source to a spacecraft in the solar wind but is refracted, scattered, or guided in the magnetosheath [Desch, 1994; Kaiser, 1998; Kaiser et al., 2004; Steinberg et al., 2004]. The higher-frequency component of the QP bursts is expected to interact with the magnetosheath the least, so direction finding analysis of the higher frequencies are more likely to show the "true" source location and not the region where the emission interacts with the magnetosheath. Furthermore, Ulysses obtained direction-finding results farther tailward when compared with the location of Cassini direction-finding results shown in Figure 16. The density of the magnetosheath should decrease tailward, allowing lower-frequency emissions generated in the magnetosphere to escape into the solar wind with minimal interaction with the magnetosheath. Thus the CassiniRPWS source locations as shown in Figure 16 do not rule the auroral region as the source of the QP bursts.

[29] Another possible source region of these emissions is the magnetospheric cusp region. Recent work by Prangé and Pallier [2003] suggested a relationship between the pulsating aurora detected by HST and the Jovian cusps at both poles. This reported relationship and the preliminary correlation between the fluctuations in the auroral UV detected by Cassini-UVIS and the weak enhancements in the trapped continuum (QP bursts) detected by Galileo [Pryor et al., 2003] suggests that reconnection events in the cusp may be a possible source of the QP emissions. Thus the preliminary Cassini direction finding results could also be consistent with a source region related to the magnetospheric cusp.

\section{Conclusion}

[30] Simultaneous observations of QP bursts by the Galileo and Cassini spacecraft show many similar characteristics, even when the two spacecraft are at very different locations and separated by large distances. These similarities suggest that the emission is beamed in a strobe light like manner over a large angular range. The observation by both spacecraft of enhancements in the trapped continuum and the dispersive shape of the emissions detected in the solar wind suggest the QP bursts interact with the higher-density magnetosheath as they propagate from the magnetosphere into the solar wind. The many other periodic events detected in the Jovian system (energetic particles, X-ray auroral hot spots, and auroral UV emissions) suggest a possible relationship with the QP radio bursts, but the lack of a clear correlation between many of the events suggests that it is not a simple relationship. The initial direction finding results from the Cassini-RPWS for one event shows a source location in the northern hemisphere, far from Jupiter. This apparent source location suggests that the lowerfrequency components of the $\mathrm{QP}$ radio burst undergoes a complex propagation process before the emissions exit into the solar wind and the apparent source location is just the exit point of the radiation. The direction-finding results may 
also suggest that the Jovian cusp region may be a possible $\mathrm{QP}$ radio burst source region.

[31] Ongoing, detailed direction finding studies with Cassini (including Cassini magnetometer, UVIS, and HST data) are examining each of the possible source locations of the QP bursts, and will attempt to (1) follow variations of the direction of arrival during individual events, examining both the variation with frequency and the evolution of the QP event with time, (2) obtain statistical results on numerous burst series, (3) measure polarization as precisely as possible, and (4) determine the source region of the Jovian QP bursts.

[32] Acknowledgments. The research at the University of Iowa is supported by the National Aeronautics and Space Administration through contract 961152 with the Jet Propulsion Laboratory. We acknowledge the support from the Iowa Cassini-RPWS team, especially Terry Averkamp. Cassini-RPWS activities at LESIA are supported by the French CNES (Centre National d'Études Spatiales). We acknowledge the support from the Meudon Cassini-RPWS team, especially Pierre Fédou and Nicole Letourneur.

[33] Arthur Richmond thanks Wayne Pryor and Andrew J. Willes for their assistance in evaluating this paper.

\section{References}

Desch, M. D. (1994), Jupiter radio bursts and particle acceleration, Astrophys. J. Suppl. Ser., 90, 541-546.

Desch, M. D., and W. M. Farrell (2000), Terrestrial LF bursts: Escape paths and wave intensification, in Radio Astronomy at Long Wavelengths, Geophys. Monogr. Ser., vol. 119, edited by R. Stone, K. Weiler, and M. Goldstein, pp. 205-211, AGU, Washington, D. C.

Gladstone, G. R., et al. (2002), A pulsating auroral X-ray hot spot on Jupiter, Nature, 415, 1000-1003.

Gurnett, D. A., W. S. Kurth, and F. L. Scarf (1983), Narrowband electromagnetic emissions from Jupiter's magnetosphere, Nature, 302, $385-$ 388

Gurnett, D. A., W. S. Kurth, R. R. Shaw, A. Roux, R. Gendrin, C. F. Kennel, F. L. Scarf, and S. D. Shawhan (1992), The Galileo plasma wave investigation, Space Sci. Rev., 60, 341-355.

Gurnett, D. A., et al. (2004), The Cassini radio and plasma wave science investigation, Space Sci. Rev., in press.

Hospodarsky, G. B., W. S. Kurth, and D. A. Gurnett (1998), Galileo observations of Jovian quasi-periodic radio bursts, paper presented at the Spring Meeting, AGU, Boston, Mass.

Kaiser, M. L. (1998), Jovian and terrestrial low frequency radio bursts: Possible cause of anomalous continuum, J. Geophys. Res., 103, 19,993-19,999.

Kaiser, M. L., M. D. Desch, W. M. Farrell, R. J. MacDowall, R. G. Stone, A. Lecacheux, B.-M. Pederson, and P. Zarka (1992), Ulysses observations of escaping VLF emissions from Jupiter, Geophys. Res. Lett., 19, 649-652.

Kaiser, M. L., P. Zarka, W. S. Kurth, G. B. Hospodarsky, and D. A. Gurnett (2000), Cassini and Wind stereoscopic observations of Jovian nonthermal radio emissions: Measurements of beam widths, J. Geophys. Res., 105, 16,053-16,062.

Kaiser, M. L., W. M. Farrell, M. D. Desch, G. B. Hospodarsky, W. S. Kurth, and D. A. Gurnett (2001), Ulysses and Cassini at Jupiter: Comparison of the quasi-periodic radio bursts, in Planetary Radio Emissions $V$, edited by
H. O. Rucker, M. L. Kaiser, and Y. Leblanc, pp. 41-48, Austrian Acad. of Sci. Press, Vienna.

Kaiser, M. L., W. M. Farrell, M. D. Desch, W. S. Kurth, G. B. Hospodarsky, and D. A. Gurnett (2004), New observations from Cassini, Ulysses, and Galileo of Jovian VLF radio emissions, J. Geophys. Res., 109, A09S08, doi:10.1029/2003JA010233.

Kurth, W. S., D. A. Gurnett, and F. L. Scarf (1989), Jovian type III radio burst, J. Geophys. Res., 94, 6917-6924.

Kurth, W. S., D. A. Gurnett, S. J. Bolton, A. Roux, and S. M. Levin (1997), Jovian radio emissions: An early overview of Galileo observations, in Planetary Radio Emissions IV, edited by H. O. Rucker, S. J. Bauer, and A. Lecacheux, pp. 1-13, Austrian Acad. of Sci. Press, Vienna.

Kurth, W. S., et al. (2002), The dusk flank of Jupiter's magnetosphere, Nature, 415, $991-994$.

MacDowall, R. J., M. L. Kaiser, M. D. Desch, W. M. Farrell, R. A. Hess, and R. G. Stone (1993), Quasiperiodic Jovian radio bursts: Observations from the Ulysses radio and plasma wave experiment, Planet. Space Sci., 41, 1059-1072.

McKibben, R. B., J. A. Simpson, and M. Zhang (1993), Impulsive bursts of relativistic electrons discovered during Ulysses' traversal of Jupiter's dusk-side magnetosphere, Planet. Space Sci., 41, 1041-1058.

Menietti, J. D., I. Christopher, and L. J. Granroth (2001), A high-resolution study of quasiperiodic radio emissions observed by the Galileo plasma wave instrument, Radio Sci., 36, 815-824.

Prangé, R., and L. Pallier (2003), Observation of Jupiter's polar cusps, paper presented at the Fall Meeting, AGU, San Francisco, Calif.

Pryor, W., G. B. Hospodarsky, I. Stewart, W. S. Kurth, L. Esposito, J. Clarke, D. Grodent, and D. A. Gurnett (2003), Cassini UVIS time-resolved Jupiter auroral data compared to QP radio bursts, paper presented at the EGSAGU-EUG Joint Asembly, Eur. Geophys. Soc., Nice, France.

Rucker, H. O., W. Macher, R. Manning, and H. P. Ladreiter (1996), Cassini model rheonetry, Radio Sci., 31, 1299-1311.

Scarf, F. L., and D. A. Gurnett (1977), A plasma wave investigation for the Voyager mission, Space Sc. Rev., 21, 289-308.

Simpson, J. A., et al. (1992a), The Ulysses cosmic ray and solar particle investigation, Astron. Astrophys. Suppl. Ser., 92, 365-399.

Simpson, J. A., et al. (1992b), Energetic charged-particle phenomena in the Jovian magnetosphere: First results from the Ulysses COSPIN collaboration, Science, 257, 1543-1550.

Steinberg, J.-L., C. Lacomb, P. Zarka, S. Hoang, and C. Perche (2004), Terrestrial low frequency bursts: Escape paths of radio waves through the bow shock, Planet. Space Sci., 52, 643-660.

Stone, R. G., et al. (1992), The unified radio and plasma wave investigation, Astron. Astrophys. Suppl. Ser., 92, 291-316.

Vogl, D. F., et al. (2004), Calibration of the Cassini-RPWS antenna system for direction-finding and polarization measurements, J. Geophys. Res., 109, A09S17, doi:10.1029/2003JA010261.

Zarka, P., B. Cecconi, and W. S. Kurth (2004), Jupiter's low frequency radio spectrum from Cassini/RPWS absolute flux density measurements, J. Geophys. Res., 109, A09S15, doi:10.1029/2003JA010260.

B. Cecconi and P. Zarka, Laboratoire d'Etudes Spatiales et d'Instrumentation en Astrophysique, Observatoire de Paris, F-92190 Meudon, France. (baptiste.cecconi@obspm.fr; phillipe.zarka@obspm.fr)

M. D. Desch and M. L. Kaiser, Laboratory for Extraterrestrial Physics, NASA Goddard Space Flight Center, Greenbelt, MD 20771, USA. (michael.desch@nasa.gov; michael.kaiser@nasa.gov)

D. A. Gurnett, G. B. Hospodarsky, and W. S. Kurth, Department of Physics and Astronomy, University of Iowa, Iowa City, IA 52232, USA. (donald-gurnett@uiowa.edu; george-hospodarsky@uiowa.edu; williamkurth@uiowa.edu) 


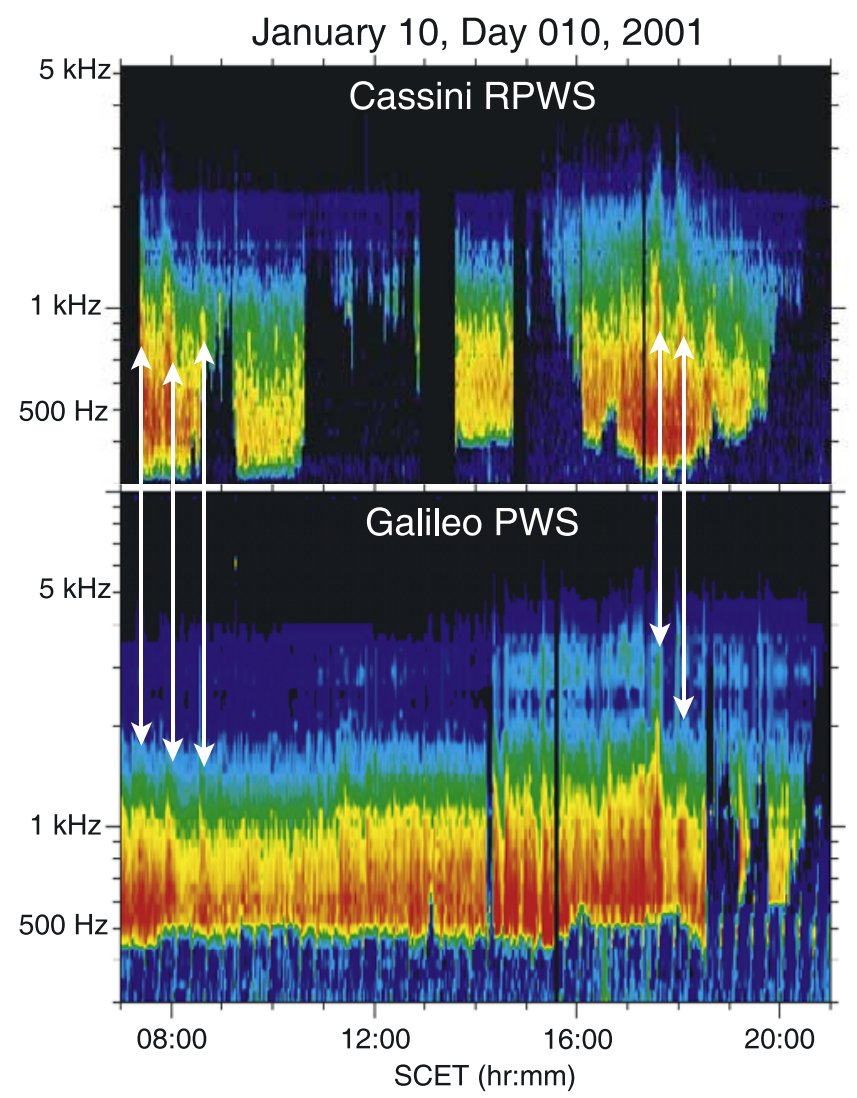

Figure 15. A frequency-time spectrogram from Cassini (top panel) and Galileo (bottom panel) from 10 January 2001, during a period when both spacecraft were located inside the Jovian magnetosphere. The data has been plotted in color to indicate amplitude with red being the most intense and blue the least intense. Arrows have been added to help point out some of the stronger enhancements in the continuum that are detected by both spacecraft. As can be seen, there is a very good agreement between the enhancements in the continuum detected by Galileo and Cassini. 